\title{
LOCALLY COMPACT SUBGROUPS OF METRIZABLE TOPOLOGICAL ABELIAN GROUPS
}

\author{
R. C. HOOPER
}

\begin{abstract}
This paper examines the question of existence of locally compact subgroups of topological abelian groups. A counterexample is given and it is shown in a certain setting that groups have a nontrivial locally compact subgroup.
\end{abstract}

1. Introduction. If $H$ is an infinite, locally compact topological abelian group, then $H$ has a nontrivial, proper locally compact subgroup $H_{1}$. More generally, suppose $H$ is an infinite, complete, metrizable, topological abelian group. We will see that $H$ may not have any nontrivial, locally compact subgroups.

When such a topological group $H$ is a subgroup of a quotient group of the Banach space $C_{0}$ modulo the integer valued sequences, $H$ will have a nontrivial locally compact subgroup. This will follow from a more general result involving Banach space with bases. The results in this paper are established by constructions utilizing coordinates.

2. A group with no nontrivial locally compact subgroups. The following corstruction will yield an example of an infinite, complete, metrizable topological group $H$ whose only locally compact subgroup is the trivial one.

Let $\left\{u_{n}\right\}_{n=1}^{\infty}$ be the standard basis for $l_{1}, v_{n}=(1 / n) u_{n}$, and $K$ be the closed subgroup of $l_{1}$ generated by $\left\{v_{n}\right\}_{n=1}^{\infty}$. Thus if $x \in K$, we can write $x$ in standard $l_{1}$ coordinates as $x=\left(p_{1} / 1, p_{2} / 2, p_{3} / 3, \ldots\right)$ with $\sum_{n=1}^{\infty}\left|p_{n}\right| / n<\infty$ and the $p_{n}$ integers. If $y \in l_{1}$ with $y=\left(a_{1}, a_{2}, \ldots\right)$, we can write $y=x+z$ with $x$ $\in K, z \in l_{1}, z=\left(\beta_{1}, \beta_{2}, \ldots\right)$ and $-1 / 2 n<\beta_{n} \leqslant 1 / 2 n$ for each $n$.

Define $L$ to be the quotient group $L=l_{1} / K$. If $w \in L, w$ is the image under the quotient map of some $y \in l_{1}$ with $y$ as above, $y=x+z$, etc. We will represent $w$ as $w=\left(\beta_{1}, \beta_{2}, \ldots\right)$ since this coordinate representation depends only on $w$.

Let $H \subset L$ be the set of $w \in L$ with $w=\left(m_{1} / 1 \cdot 2, m_{2} / 2 \cdot 2^{2}, m_{3} / 3\right.$ $\left.\cdot 2^{3}, \ldots\right)$, each $m_{n}$ an integer, $\quad-2^{n-1}<m_{n} \leqslant 2^{n-1}$, and $m_{n+1}$ $\equiv m_{n}\left(\bmod 2^{n}\right) . H$ is a closed subgroup of $L$ and in fact is a subgroup of a projective limit group, the 2 -adic integers, but has a different topology inherited from $L$. If $w$ and the $m_{n}$ are as above, define a function $\sigma$ on $H$ by

Received by the editors July 1, 1975.

AMS (MOS) subject classifications (1970). Primary 22A05; Secondary 46B99.

Key words and phrases. Topological group, locally compact subgroup, closed subgroups of Banach spaces. 
$\sigma(w)=\left(m_{1}, m_{2}, \ldots\right)$. Define for $w \in H,\|w\|=\sum_{n=1}^{\infty}\left|m_{n}\right| / n 2^{n}$.

Define $H_{n}=\left\{w \in H \mid w=\left(\beta_{1}, \beta_{2}, \ldots\right)\right.$ with $\beta_{i}=0$ if $\left.i \leqslant n\right\}$. Then the $H_{n}$ are open subgroups of $H$ and the intersection of all the $H_{n}$ is the identity.

If $w \in H$ with $\sigma(w)=\left(m_{1}, m_{2}, \ldots\right)$, then for each $n, m_{n+1}=m_{n}$ $+a_{n} 2^{n}$ with $a_{n}=0,1$ or -1 . Define $a_{0}=m_{1}$. Then $m_{n+1}=\sum_{r=0}^{n} a_{r} 2^{r}$. Note that if $r$ is the least integer with $a_{r} \neq 0$, then $a_{r}=1$ since $m_{r+1}$ cannot be $-2^{r}$ by definition of the coefficients $m_{n}$.

This induces a function $\theta$ on $H$ defined by $\theta(w)=\left(a_{0}, a_{1}, \ldots\right)$ for $w \in H$. If $\theta(w)=\left(a_{0}, a_{1}, \ldots\right)$ define $\|w\|^{\prime}$ by $\|w\|^{\prime}=\sum_{r=0}^{\infty}\left|a_{r}\right| /(r+1)$. Then if $\sigma(w)$ $=\left(m_{1}, m_{2}, \ldots\right)$,

$$
\begin{aligned}
\|w\| & =\sum_{n=1}^{\infty} \frac{\left|m_{n}\right|}{n 2^{n}}=\sum_{n=1}^{\infty} \frac{1}{n 2^{n}}\left|\sum_{r=0}^{n-1} a_{r} 2^{r}\right| \leqslant \sum_{n=1}^{\infty} \frac{1}{n 2^{n}} \sum_{r=0}^{n-1}\left|a_{r}\right| 2^{r} \\
& =\sum_{r=0}^{\infty}\left|a_{r}\right| 2^{r} \sum_{n=r+1}^{\infty} \frac{1}{n 2^{n}}=\sum_{r=0}^{\infty}\left|a_{r}\right| \sum_{n=r+1}^{\infty} \frac{1}{n 2^{n-r}} \\
& =\sum_{r=0}^{\infty}\left|a_{r}\right| \sum_{k=1}^{\infty} \frac{1}{k+r} \frac{1}{2^{k}} \leqslant \sum_{r=0}^{\infty} \frac{\left|a_{r}\right|}{r+1} \sum_{k=1}^{\infty} \frac{1}{2^{k}}=\sum_{r=0}^{\infty} \frac{\left|a_{r}\right|}{r+1}=\|w\|^{\prime} .
\end{aligned}
$$

Thus,

$$
\|w\| \leqslant\|w\|^{\prime}
$$

On the other hand,

$$
\begin{aligned}
\|w\|^{\prime} & =\sum_{r=0}^{\infty} \frac{\left|a_{r}\right|}{r+1} \leqslant 2 \sum_{r=0}^{\infty}\left|a_{r}\right| \sum_{k=1}^{\infty} \frac{1}{r+k} \frac{1}{2^{k}} \\
& =2 \sum_{r=0}^{\infty}\left|a_{r}\right| \sum_{n=r+1}^{\infty} \frac{1}{n} \frac{1}{2^{n-r}}=2 \sum_{r=0}^{\infty}\left|a_{r}\right| 2^{r} \sum_{n=r+1}^{\infty} \frac{1}{n 2^{n}} \\
& =2 \sum_{n=1}^{\infty} \frac{1}{n 2^{n}} \sum_{r=0}^{n-1}\left|a_{r}\right| 2^{r} .
\end{aligned}
$$

Thus,

$$
\|w\|^{\prime} \leqslant 2 \sum_{n=1}^{\infty} \frac{1}{n 2^{n}} \sum_{r=0}^{n-1}\left|a_{r}\right| 2^{r} .
$$

Suppose $m_{p+1} \neq 0$ and let $q$ be the greatest $q$ with $q \leqslant p$ and $a_{q} \neq 0$. Then

$$
\left|m_{p+1}\right|=\left|a_{q} 2^{q}+m_{q}\right| \geqslant\left|a_{q} 2^{q}\right|-\left|m_{q}\right| \geqslant 2^{q}-2^{q-1}=2^{q-1} .
$$

This implies

$$
\sum_{r=0}^{p}\left|a_{r}\right| 2^{r}=\sum_{r=0}^{q}\left|a_{r}\right| 2^{r} \leqslant \sum_{r=0}^{q} 2^{r} \leqslant 2^{q+1} \leqslant 4\left|m_{p+1}\right| .
$$

Combining (2.2) with (2.3) gives

$$
\|w\|^{\prime} \leqslant 2 \sum_{n=1}^{\infty} \frac{4\left|m_{n}\right|}{n 2^{n}}=8\|w\|
$$


Combining this with $(2.1)$ we get $\frac{1}{8}\|w\|^{\prime} \leqslant\|w\| \leqslant\|w\|^{\prime}$. Whence \|\| and \|\|$^{\prime}$ yield the same topology on $H$.

Let $w \in H, w \neq 0$, and $\theta(w)=\left(a_{0}, a_{1}, \ldots\right)$. If $k$ is an integer with $0 \leqslant k$ $<n$, then

$$
2^{k} m_{n}=2^{k} \sum_{r=0}^{n-1} a_{r} 2^{r}=\sum_{r=0}^{n-1} a_{r} 2^{k+r} \equiv \sum_{r=0}^{n-k-1} a_{r} 2^{k+r} \quad\left(\bmod 2^{n}\right) .
$$

Choose $p$ to be the least integer with $a_{p} \neq 0$. Define $s$ so that $s a_{p}=\left|a_{p}\right|$. Thus if $y=2^{k} w$, then $\theta(y)=\left(b_{0}, b_{1}, \ldots\right)$ with $b_{j}=0$ if $j<k$ and $b_{j}=s a_{j-k}$ if $j$ $\geqslant k$. Whence

$$
\|y\|^{\prime}=\sum_{j=0}^{\infty} \frac{\left|b_{j}\right|}{j+1}=\sum_{j=k}^{\infty} \frac{\left|b_{j}\right|}{j+1}=\sum_{j=k}^{\infty} \frac{\left|s a_{j-k}\right|}{j+1}=\sum_{r=0}^{\infty} \frac{\left|a_{r}\right|}{r+k+1} .
$$

Given $\epsilon>0$, choose $N$ so that $\sum_{r=N}^{\infty}\left|a_{r}\right| /(r+1)<\epsilon / 2$. Choose $t$ to be a positive integer with $1 / t<\epsilon / 2$. If $k \geqslant N t$, then

$$
\|y\|^{\prime}=\sum_{r=0}^{N-1} \frac{\left|a_{r}\right|}{r+k+1}+\sum_{r=N}^{\infty} \frac{\left|a_{r}\right|}{r+k+1}<\frac{N}{N t}+\frac{\epsilon}{2}<\epsilon .
$$

This proves if $w \in H$ with $w \neq 0$, then the subgroup generated by $w$ is not discrete.

Again suppose $w \in H, w \neq 0$, and let $H_{1}$ be the closed subgroup generated by $w$. Suppose $\theta(w)=\left(a_{0}, a_{1}, \ldots\right)$ and $p$ is the least integer with $a_{p} \neq 0$. Given an integer $n>p$, choose $s$ with $s=-1$ if $p$ is odd and $s=1$ if $p$ is even, and choose an integer $q_{n}$ so that

$$
q_{n} \sum_{r=p}^{n-1} a_{r} 2^{r} \equiv s \sum_{k=p}^{n-1}(-1)^{k} 2^{k} \quad\left(\bmod 2^{n}\right) .
$$

If $y_{n}=q_{n} w$, then $\theta\left(y_{n}\right)=\left(b_{0}, b_{1}, \ldots\right)$ with $b_{k}=s(-1)^{k}$ if $p \leqslant k<n$ and $b_{k}=0$ if $k<p$. Thus $\left\|y_{n}\right\|^{\prime} \geqslant \sum_{k=p}^{n-1} 1 /(k+1)$.

If $H_{1}$ is compact, then $\left\{y_{n}\right\}_{n=1}^{\infty}$ has a subsequence converging to some $y \in H_{1}$. But $\lim _{n \rightarrow \infty}\left\|y_{n}\right\|^{\prime}=\infty$ which contradicts $\|y\|^{\prime}<\infty$.

Whence the only subgroup $F$ of $H$ such that $F$ is discrete or compact is the trivial subgroup. By the structure theorem for locally compact abelian groups, it follows that the only locally compact subgroup of $H$ is the trivial subgroup. $H$ does have some nontrivial, proper subgroups, e.g., the $H_{n}$.

This example leaves unanswered the question of whether, if $H$ is an infinite, complete, metrizable topological abelian group, $H$ has a nontrivial, proper closed subgroup.

3. Some groups which have nontrivial locally compact subgroups. If $C_{0}$ is the Banach space of real sequences which converge to zero and $K$ the group of integer sequences with only a finite number of nonzero terms, define $G$ to be the quotient group $C_{0} / K$ [1]. If $H$ is a nontrivial, closed subgroup of $G$, then we will see that $H$ has a nontrivial locally compact subgroup. This will be a consequence of a more general result for Banach spaces which have a basis.

Let $\left\{u_{n}\right\}_{n=1}^{\infty}$ be a basis for a Banach space $\left(E,\|\|^{\prime \prime}\right)$ with $\left\|u_{n}\right\|^{\prime \prime}=1$ for all 
$n$. There is a norm \|\|$^{\prime}$ on $E$ equivalent to \|\|$^{\prime \prime}$ such that

$$
\left\|\sum_{n=1}^{\infty} a_{n} u_{n}\right\|^{\prime}=\sup _{m, p}\left\|\sum_{n=m}^{p} a_{n} u_{n}\right\|^{\prime \prime}
$$

if $\sum_{n=1}^{\infty} a_{n} u_{n} \in E\left[2\right.$, p. 180, Theorem 19.1(c)]. By (3.1), $\left\|u_{n}\right\|^{\prime}=1$. In general, if $\sum_{n=1}^{\infty} a_{n} u_{n} \in E$, then

$$
\begin{aligned}
\left\|\sum_{n=1}^{\infty} a_{n} u_{n}\right\|^{\prime} & =\sup _{m, p}\left\|\sum_{n=m}^{p} a_{n} u_{n}\right\|^{\prime \prime}=\sup _{m, p}\left(\sup _{m \leqslant k \leqslant l \leqslant p}\left\|\sum_{n=k}^{l} a_{n} u_{n}\right\|^{\prime \prime}\right) \\
& =\sup _{m, p}\left\|\sum_{n=m}^{p} a_{n} u_{n}\right\|^{\prime} .
\end{aligned}
$$

Thus,

$$
\left\|\sum_{n=1}^{\infty} a_{n} u_{n}\right\|^{\prime}=\sup _{m, p}\left\|\sum_{n=m}^{p} a_{n} u_{n}\right\|^{\prime} .
$$

Let $K=\left\{x \in E \mid x=\sum_{n=1}^{\infty} s_{n} u_{n}\right.$ with $\left.s_{n} \in Z\right\}$. $K$ is a discrete subgroup since (3.1) implies if $x \neq 0$ and $x=\sum_{n=1}^{\infty} s_{n} u_{n}$ with $s_{n} \in Z$, then $\|x\|^{\prime}$ $\geqslant \max _{n}\left\|s_{n} u_{n}\right\|^{\prime \prime} \geqslant 1$. Also, if $x=\sum_{n=1}^{\infty} a_{n} u_{n}$, then $\lim _{n \rightarrow \infty} a_{n}=0[2$, p. 20 , Lemma 3.1]. Thus if $x=\sum_{n=1}^{\infty} s_{n} u_{n}$ is in $K$, then $s_{n} \in Z$ and for some $N, x=\sum_{n=1}^{N} s_{n} u_{n}$.

Let $G$ be the quotient group $G=E / K$ and $\pi: E \rightarrow G$ be the quotient map. Define $\|y\|$ for $y \in G$ to be the minimum of $\|x\|^{\prime}$ over all $x \in E$ with $\pi(x)$ $=y$. If $x \in E$, we can write $x=\sum_{n=1}^{\infty} x_{n} u_{n}+w$ with $w \in K, x_{n}$ $\in R$ and $-\frac{1}{2}<x_{n} \leqslant \frac{1}{2}$. If $y=\pi(x)$, we will write $y=\left(y_{1}, y_{2}, \ldots\right)$ with $y_{n}$ $=x_{n}$ for the $x_{n}$ just described. In general, if $y, z \in G$, then $\|y+z\|$ $\leqslant\|y\|+\|z\|$ and $\|y\|=\|-y\|$.

LemMa . Suppose $H$ is a closed subgroup of $G$. Also suppose that, given a positive integer $n>1$ and positive real numbers $\epsilon$ and $M$, there is $b=\left(b_{1}\right.$, $\left.b_{2}, \ldots\right)$ in $H$ with $0<\|b\| \leqslant \epsilon$ and with $\left\|\left(b_{1}, b_{2}, \ldots, b_{n}, 0,0, \ldots\right)\right\| \leqslant M\|b\|$. Then $H$ contains an infinite, discrete subgroup.

Proof. Choose $b^{1}=\left(b_{1}^{1}, b_{2}^{1}, \ldots\right)$ in $H$ such that $0<\left\|b^{1}\right\| \leqslant \frac{1}{2}$. Then for some positive integer $r, \frac{1}{4}<|r|\left\|b^{1}\right\| \leqslant \frac{1}{2}$. Define $c^{1}=r b^{1}$. Write $c^{1}=\left(c_{1}^{1}\right.$, $\left.c_{2}^{1}, \ldots\right)$. Define $k(1)=0$ and choose $k(2)>0$ so that

$$
\left\|\left(0,0, \ldots, 0, c_{k(2)+1}^{1}, c_{k(2)+2}^{1}, \ldots\right)\right\|<1 / 2 \cdot 16 \cdot 2^{2} .
$$

Inductively suppose $\left\{c^{m}\right\}_{m=1}^{n} \subset H$ and $\{k(m)\}_{m=1}^{n+1}$ are given such that $m_{1}$ $<m_{2}$ implies $k\left(m_{1}\right)<k\left(m_{2}\right), 1 / 2 \cdot 2^{m}<\left\|c^{m}\right\| \leqslant 1 / 2^{m}, m<m_{1} \leqslant n+1$ implies

$$
\left\|\left(0,0, \ldots, 0, c_{k\left(m_{1}\right)+1}^{m}, c_{k\left(m_{1}\right)+2}^{m}, \ldots\right)\right\|<1 / m_{1} \cdot 16 \cdot 2^{m_{1}},
$$

and that $m \leqslant n$ implies

$$
\left\|\left(c_{1}^{m}, c_{2}^{m}, \ldots, c_{k(m)}^{m}, 0,0, \ldots\right)\right\|<1 / 2^{2 m} \cdot 16 .
$$


By the hypothesis of the lemma, we can find $b^{n+1}=\left(b_{1}^{n+1}, b_{2}^{n+1}, \ldots\right)$ in $H$ with $0<\left\|b^{n+1}\right\| \leqslant 1 / 2^{n+1}$ and

$$
\left\|\left(b_{1}^{n+1}, b_{2}^{n+1}, \ldots, b_{k(n+1)}^{n+1}, 0,0, \ldots\right)\right\| \leqslant\left\|b^{n+1}\right\| / 2^{n+1} \cdot 16 .
$$

Choose a positive integer $r$ so that $1 / 2 \cdot 2^{n+1}<|r|\left\|b^{n+1}\right\| \leqslant 1 / 2^{n+1}$. Define $c^{n+1}=r b^{n+1}$. Choose $k(n+2)$ so that $k(n+1)<k(n+2)$ and $m<n+2$ implies

$$
\left\|\left(0,0, \ldots, 0, c_{k(n+2)+1}^{m}, c_{k(n+2)+2}^{m}, \ldots\right)\right\|<1 /(n+2) \cdot 16 \cdot 2^{n+2} .
$$

Note that

$$
\left\|\left(0,0, \ldots, 0, c_{k(n)+1}^{n}, c_{k(n)+2}^{n}, \ldots, c_{k(n+1)}^{n}, 0,0, \ldots\right)\right\| \geqslant \frac{1}{2}\left\|c^{n}\right\|
$$

for all $n$.

Define $a \in H$ by $a=\sum_{p=1}^{\infty} c^{p} \cdot \sum_{p=1}^{\infty} c^{p}$ makes sense since $\sum_{p=1}^{\infty}\left\|c^{p}\right\|$ $\leqslant \sum_{p=1}^{\infty} 1 / 2^{p}=1$ and since $H$ is complete. Write $a=\left(a_{1}, a_{2}, \ldots\right)$.

Given an integer $q \neq 0$, choose $n \geqslant 0$ so that $2^{n} \leqslant q<2^{n+1}$. Then by (3.2), the definition of $K$ and \|\| , we get

$$
\begin{aligned}
\|q a\| \geqslant & \left\|q\left(0, \ldots, 0, a_{k(n+2)+1}, \ldots, a_{k(n+3)}, 0,0, \ldots\right)\right\| \\
\geqslant|q| & \left(\left\|\left(0, \ldots, 0, c_{k(n+2)+1}^{n+2}, \ldots, c_{k(n+3)}^{n+2}, 0,0, \ldots\right)\right\|\right. \\
& \quad-\sum_{m=1}^{n+1}\left\|\left(0, \ldots, 0, c_{k(n+2)+1}^{m}, \ldots, c_{k(n+3)}^{m}, 0,0, \ldots\right)\right\| \\
& \left.\quad-\sum_{m=n+3}^{\infty}\left\|\left(0, \ldots, 0, c_{k(n+2)+1}^{m}, \ldots, c_{k(n+3)}^{m}, 0,0, \ldots\right)\right\|\right) \\
\geqslant & 2^{n}\left(\frac{1}{2} \cdot \frac{1}{2 \cdot 2^{n+2}}-\sum_{m=1}^{n+1} \frac{1}{n+2} \cdot \frac{1}{16 \cdot 2^{n+2}}-\sum_{m=n+3}^{\infty} \frac{1}{2^{2 m} \cdot 2}\right) \\
\geqslant & 1 / 16-1 / 64-1 / 64=1 / 16-1 / 32=1 / 32 .
\end{aligned}
$$

Thus $a$ generates a discrete subgroup of $H$.

THEOREM. Let $\left(E,\|\|^{\prime}\right)$ be a Banach space with a basis $\left\{u_{n}\right\}_{n=1}^{\infty}$ such that $\left\|u_{n}\right\|^{\prime}=1$ for all $n$. Also let $K$ be the subgroup generated by $\left\{u_{n}\right\}_{n=1}^{\infty}$ and $G$ $=E / K$. If $H$ is a nontrivial, closed subgroup of $G$, then either $H$ has a nontrivial, compact subgroup or $H$ has an infinite cyclic, discrete subgroup.

Proof. Suppose $H$ is a nontrivial, closed subgroup of $G$ whose only discrete subgroup is the trivial group. Then by the lemma, there is an integer $n>0$ and real numbers $\epsilon>0$ and $M>0$ such that there is no $b=\left(b_{1}, b_{2}, \ldots\right)$ in $H$ with $0<\|b\| \leqslant \epsilon$ and $\left\|\left(b_{1}, \ldots, b_{n}, 0, \ldots\right)\right\| \leqslant M\|b\|$.

Define $L=\left\{x \in G \mid x=\left(x_{1}, x_{2}, \ldots\right)\right.$ and $x_{i}=0$ if $\left.i>n\right\}$ and define $\theta$ : $G$ $\rightarrow L$ to be the projection with $\theta(y)=\left(z_{1}, z_{2}, \ldots\right)$ for $y=\left(y_{1}, y_{2}, \ldots\right), z_{i}$ $=y_{i}$ if $i \leqslant n$ and $z_{i}=0$ if $i>n$. Also define $U=\{x \in H \mid\|x\| \leqslant \epsilon / 2\}$.

Suppose $\left\{x^{i}\right\}_{i=1}^{\infty}$ is in $U$ and $\left\{\theta\left(x_{i}\right)\right\}_{i=1}^{\infty}$ is a nonrepeating Cauchy sequence 
in $L$. Then if $\epsilon_{1}>0$ is given, there is $N\left(\epsilon_{1} M\right)$ such that $i, j>N\left(\epsilon_{1} M\right)$ implies $\left\|\theta\left(x^{i}\right)-\theta\left(x^{j}\right)\right\|<\epsilon_{1} M$. But we know since $\left\|x^{i}-x^{j}\right\| \leqslant \epsilon$ that $\left\|\theta\left(x^{i}-x^{j}\right)\right\|$ $\geqslant M\left\|x^{i}-x^{j}\right\|$. Thus, $\epsilon_{1} M>\left\|\theta\left(x^{i}\right)-\theta\left(x^{j}\right)\right\| \geqslant M\left\|x^{i}-x^{j}\right\|$ and $\left\|x^{i}-x^{j}\right\|$ $<\epsilon_{1}$ so that $\left\{x^{i}\right\}_{i=1}^{\infty}$ is a Cauchy sequence in $U$.

If $\left\{y^{j}\right\}_{j=1}^{\infty}$ is any sequence in $U$, then $\left\{\theta\left(y^{j}\right)\right\}_{j=1}^{\infty}$ has a convergent subsequence $\left\{\theta\left(y^{j(i)}\right)\right\}_{i=1}^{\infty}$ since $L$ is compact. The preceding result shows $\left\{y^{j(i)}\right\}_{i=1}^{\infty}$ is a Cauchy sequence in $U . U$ is a complete metric space under the metric induced from \|\| since $G=E / K$ is complete, $H$ is closed in $G$, and $U$ is closed in $H$. Whence $\left\{y^{j(i)}\right\}_{i=1}^{\infty}$ converges to some $y \in U$. This proves that $U$ is compact. Whence $H$ is a locally compact topological group. Then the structure theorem for locally compact abelian groups implies $H$ has an infinite cyclic, discrete subgroup or a nontrivial compact subgroup.

It is not known whether this theorem holds true if the hypotheses of the theorem are weakened so that $E$ is any separable Banach space and $K$ is a discrete subgroup of $E$.

Let $E$ be a separable Banach space and $K$ a discrete subgroup. $K$ is said to have the maximal property if $K_{1}$ is a discrete subgroup and $K_{1} \supset K$ imply that $K_{1} / K$ is a torsion group. If $K$ is a discrete subgroup of a Banach space $E$ such that $K$ does not have the maximal property, then $E / K$ has an infinite, discrete subgroup. If $E$ is finite dimensional of dimension $n$, then any set of $n$ elements which are linearly independent over the reals generate a subgroup $K$ with the maximal property. No example is known of such a pair $E, K$ if $E$ is infinite dimensional.

\section{REFERENCES}

1. S. Rolewicz, Some remarks on monothetic groups, Colloq. Math. 13 (1964), 27-28. MR 30 \#2102.

2. I. Singer, Bases in Banach spaces. I, Die Grundlehren der math. Wissenschaften, Band 154, Springer-Verlag, New York and Berlin, 1970. MR 45 \#7451.

Department of Mathematics, University of Nevada, Reno, Nevada 89507 